\title{
Multiple genome viewer (MGV): a new tool for visualization and comparison of multiple annotated genomes
}

\author{
Joel E. Richardson ${ }^{1}$ (D) $\cdot$ Richard M. Baldarelli $^{1}$ (D) Carol J. Bult ${ }^{1}$ (D)
}

Received: 5 July 2021 / Accepted: 8 August 2021 / Published online: 27 August 2021

(c) The Author(s) 2021

\begin{abstract}
The assembled and annotated genomes for 16 inbred mouse strains (Lilue et al., Nat Genet 50:1574-1583, 2018) and two wild-derived strains (CAROLI/EiJ and PAHARI/EiJ) (Thybert et al., Genome Res 28:448-459, 2018) are valuable resources for mouse genetics and comparative genomics. We developed the multiple genome viewer (MGV; http://www.informatics. jax.org/mgv) to support visualization, exploration, and comparison of genome annotations within and across these genomes. MGV displays chromosomal regions of user-selected genomes as horizontal tracks. Equivalent features across the genome tracks are highlighted using vertical 'swim lane' connectors. Navigation across the genomes is synchronized as a researcher uses the scroll and zoom functions. Researchers can generate custom sets of genes and other genome features to be displayed in MGV by entering genome coordinates, function, phenotype, disease, and/or pathway terms. MGV was developed to be genome agnostic and can be used to display homologous features across genomes of different organisms.
\end{abstract}

\section{Introduction}

The availability of multiple annotated mouse genome assemblies serves as a foundation for investigating the genomic and genetic basis for phenotype diversity across inbred and wild-derived strains of laboratory mice (Keane et al. 2011; Lilue et al. 2018; Thybert et al. 2018). However, even with the availability of widely used genome browsers, interactive and customizable comparison of annotated genome features and their organization across multiple genomes remains a significant barrier to accessing and exploring these genomes. Here we describe the multiple genome viewer (MGV) which we developed to support interactive exploration and visualization of genome annotations for multiple lines of laboratory mice and for easy access to the sequence data for these annotations for alignment and data analysis. MGV relies on the mouse genome assemblies available from the Ensembl genome browser. The genome feature annotations displayed in MGV come from the mouse genome database (MGD) unified gene catalog (Zhu et al. 2015). The MGD unified gene catalog combines genome feature predictions from Ensembl/ GenCode and NCBI into a single non-redundant annotation

Carol J. Bult

carol.bult@jax.org

1 The Jackson Laboratory, Bar Harbor, ME, USA set which serves as the foundation for the expertly curated phenotype, function, and developmental expression annotations for mouse genes available from MGD and the gene expression database (GXD) (Baldarelli et al. 2021; Blake et al. 2020; Zhu et al. 2015) Although the implementation for MGV reported here is focused on mouse genomes, the software is genome agnostic and was designed to accommodate multiple annotated genomes from any organism and can also support the display of homologous genome features across different organisms.

To represent genome features of multiple strains, MGD uses the concept of a canonical genome feature (Fig. 1A). A canonical genome feature is one that exists in any strain or species of Mus. In MGD, any genome feature that has a unique, permanent MGI accession id is a canonical feature. Canonical gene records are linked to instances of the genome feature found in the genome of different Mus strains and species. The canonical genome feature concept supports rapid identification of strain-specific genome features and the capacity to associate biological annotations with specific strains. MGD provides a tabular summary of the strain distribution of genome features along with their strain-specific identifiers and genome coordinates (Fig. 1B). 


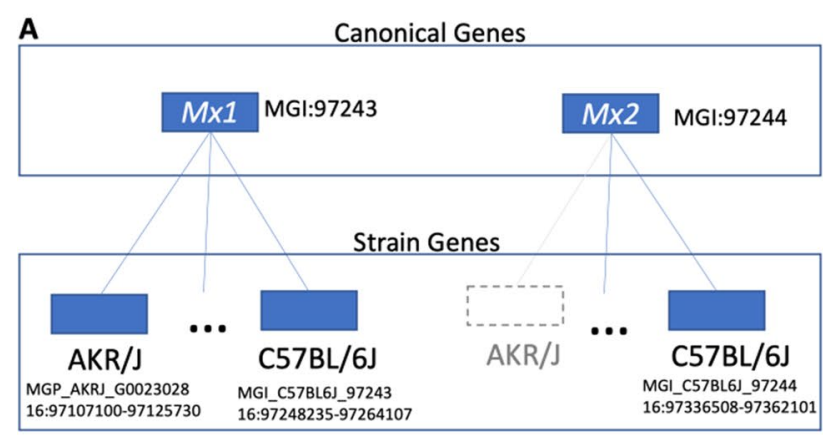

Fig. 1 Canonical genes are the abstract representations of any/all identified mouse genome features regardless of strain. A Canonical genes have MGI accession ids and official nomenclature and historically have been the entities associated with biological annotations about function, phenotype, and disease. With the advent of multiple mouse genome assemblies, the concept of a strain gene was added to support robust representation of strain-specific genome annotations and attributes. Strain genes have strain-specific accession identifiers

\section{Results}

\section{MGV's user interface}

The user interface for MGV consists of a control panel, tracks for displaying genome feature maps of user-selected genomes, and a genome-wide overview (Fig. 2). The control panel on the left side of the display comprises several modules that allow users to control which genomes and features to display, options to modify display properties, controls for downloading sequences, and the ability to create and display lists of features. The basic functions for MGV are described below. The MGV user guide lists the key and mouse driven commands for the interface and can be invoked at any time while using the software with the $\mathrm{H}$ command.

\section{User interaction}

The user interacts with MGV with both the mouse and with keyboard commands. For example, dragging on a displayed region scrolls the region, while pressing the 'H' key opens or closes the Help screen. Many commands have both mouse- and key-based versions, e.g., the left and right arrow keys can also be used to scroll. MGV uses key combinations to modify commands. For example, holding down the shift key while dragging (shift-drag) zooms into the specified region. Note that the "option" key is "alt" on some keyboards; likewise, the "command" key
B

\begin{tabular}{|c|c|c|c|}
\hline Strain & Gene Model ID & Feature Type & Coordinates \\
\hline C57BL/6] & MGI_C57BL6]_97244 & polymorphic pseudogene & Chr16:97336508-97362101 (+) \\
\hline $129 \mathrm{~S} 1 / \mathrm{SvImJ}$ & MGP_129S1SvImJ_G0009937 & polymorphic pseudogene & Chr16:98975321-99001926(+) \\
\hline $\mathrm{A} / \mathrm{J}$ & MGP_AJ_G0009921 & polymorphic pseudogene & Chr16:94306282-94333178 (+) \\
\hline AKR/J & no annotation & & \\
\hline $\mathrm{BALB} / \mathrm{cl}$ & MGP_BALBCI_G0009906 & polymorphic pseudogene & Chr16:94852897-94879920 (+) \\
\hline $\mathrm{C} 3 \mathrm{H} / \mathrm{HeJ}$ & MGP_C3HHeJ_G0009838 & polymorphic pseudogene & Chr16:97732722-97759089 (+) \\
\hline C57BL/6NJ & MGP_C57BL6NJ_G0010152 & polymorphic pseudogene & Chr16:102065499-102096467 (+) \\
\hline CAROLI/EIJ & MGP_CAROLIEIJ_G0008949 & polymorphic pseudogene & Chr16:92139309-92168414 (+) \\
\hline CAST/EIJ & MGP_CASTEIJ_G0009656 & polymorphic pseudogene & Chr16:98540562-98573150 (+) \\
\hline $\mathrm{CBA} / \mathrm{J}$ & no annotation & & \\
\hline $\mathrm{DBA} / 2 \mathrm{~J}$ & MGP_DBA2J_G0009839 & polymorphic pseudogene & Chr16:94165560-94193426 (+) \\
\hline $\mathrm{FVB} / \mathrm{NJ}$ & MGP_FVBNJ_G0009856 & polymorphic pseudogene & Chr16:93326378-93353473 (+) \\
\hline LP/] & MGP_LP]_G0009934 & polymorphic pseudogene & Chr16:98621960-98651094 (+) \\
\hline NOD/ShiltJ & MGP_NODShiLt]_G0009823 & polymorphic pseudogene & Chr16:110647208-110674730(+) \\
\hline NZO/HILt] & MGP_NZOHILt__G0010125 & polymorphic pseudogene & Chr16:97756736-97786036(+) \\
\hline $\mathrm{PWK} / \mathrm{PhJ}$ & MGP_PWKPhJ_G0009577 & polymorphic pseudogene & Chr16:94506985-94522251 (+) \\
\hline SPRET/EiJ & no annotation & & \\
\hline WSB/Ei] & MGP_WSBEIJ_G0009758 & polymorphic pseudogene & Chr16:98025523-98054325 (+) \\
\hline
\end{tabular}

and genome coordinates. A given canonical mouse gene may or may not exist in a specific mouse strain, as shown for Mx2 (MGI:97244), which has not been annotated in the AKR/J inbred strain. B The strain distribution table for the $M x 2$ gene showing the strain-specific identifiers and genome locations. This table is available from the gene detail page in MGD. The $M x 2$ gene is a polymorphic pseudogene meaning the gene codes for a functional protein in some strains, but not others

is sometimes "meta". The Help screen lists all the mouse and key commands.

\section{Genome selection}

Any number of genomes can be selected for display from a list of those available. For the laboratory mouse, preselected groups of genomes- including the eight founder strains of the Collaborative Cross (Iraqi et al. 2008) are also available. In addition to comparing annotations across different mouse genomes, MGV can be used to compare the annotations of different assembly versions of the same genome. For example, MGV includes annotated genomes for both GRCm38 and the most recent GRCm39 assemblies of the C57BL/6J reference genome. To support comparisons of orthologous genome features across different organisms, MGV includes annotated reference genome assemblies of human (Homo sapiens), fruit fly (Drosophila melanogaster), zebrafish (Danio rerio), nematode (Caenorhabditi elegans), rat (Rattus norvegicus), and yeast (Saccharomyces cerevisiae). Annotations for these genomes and the definitions of orthologous genome features are obtained from the Alliance of Genome Resources (Alliance of Genome Resources 2019; Alliance of Genome Resources Consortium 2019).

By design, MGV has no predefined reference genome; any genome can be selected as the reference, which then determines which regions are shown for other displayed genomes. Selecting a reference genome is optional; however, other modes support aligning the genomes around common 


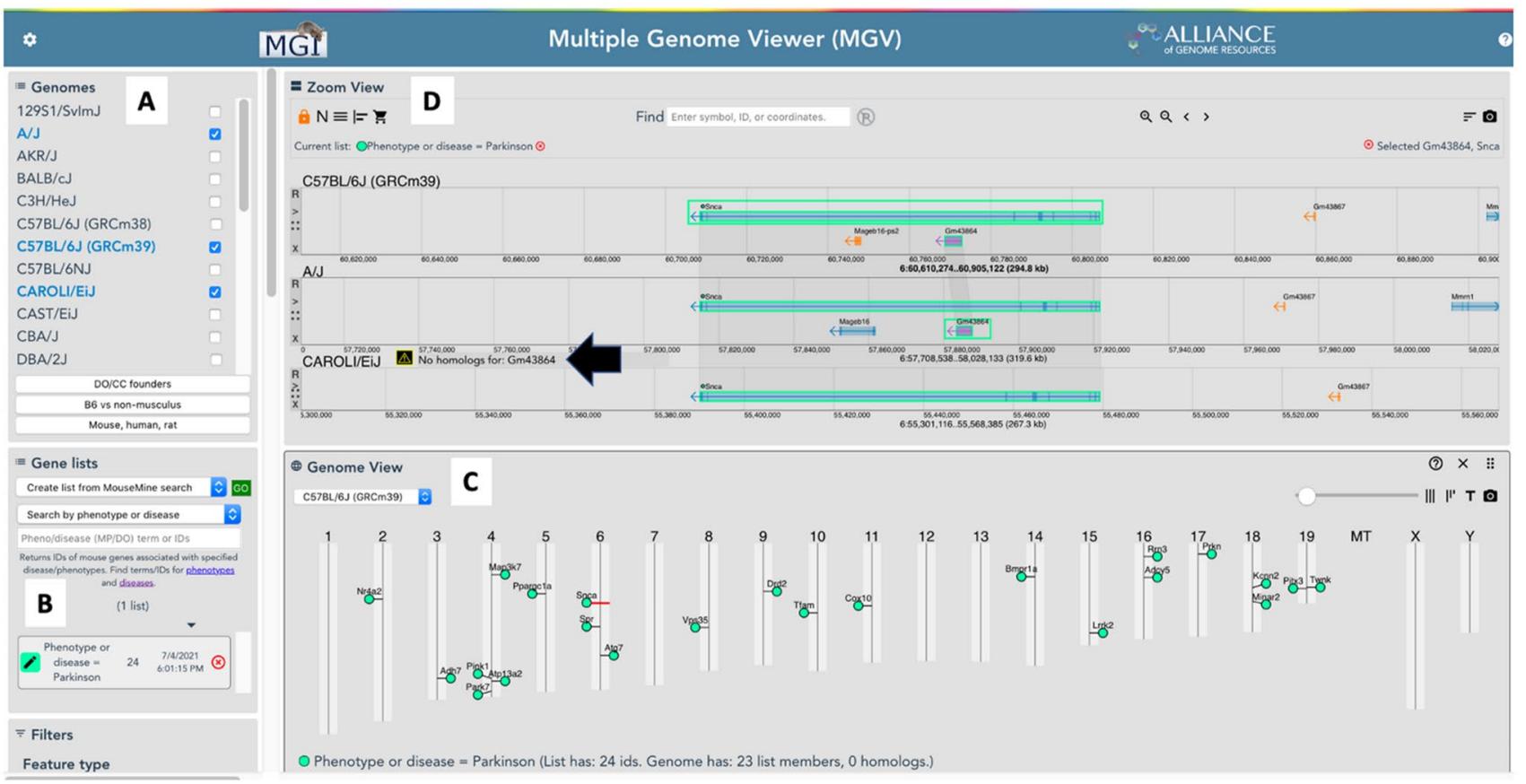

Fig. 2 Screenshot of the MGV user interface showing results of a Gene List search for mouse genes associated with Parkinson's disease. Note: not all display function modules are shown in this figure. A The genomes module allows users to select which genome tracks to display. Here, C57BL/6J, A/J, and CAROLI/EiJ have been selected. B Users can search for sets of mouse genes by various attributes. Here, the search was for genes in the mouse genome database (MGD) annotated to the disease term, Parkinson's disease. $\mathbf{C}$ The results of the

landmarks, or even using each displayed genome as an independently scrollable and zoomable browser.

\section{Feature selection}

Genome features are selected by clicking on them. Multiple features can be selected by pressing the shift key while clicking. Selected features are highlighted and may show other differences depending on other control settings. For example, one can use the contrast slider (under settings) to fade unselected features, or the expand setting to show the transcripts of selected features (or of all features).

\section{Display filters}

The genome features displayed in MGV can be toggled on and off according to multiple criteria, including feature biotype (i.e., protein coding, pseudogene, etc.), feature length, features present in some displayed genomes but not others, and features that have been selected by the user. gene set search for Parkinson's disease are automatically displayed on the genome map. D Swim lane connectors highlight equivalent features across the genome tracks. Here, the Snca gene (MGI:1277151) is highlighted showing its location across the three user-selected genomes. Gene, Gm43864 (MGI:5690256), is only annotated to the $\mathrm{C} 57 \mathrm{BL} / 6 \mathrm{~J}$ and $\mathrm{A} / \mathrm{J}$ genomes. A message is displayed on the CAROLI/ EiJ track to indicate that $G m 43864$ is not found in that genome (black arrow)

\section{Display controls}

MGV provides several tools for modifying display attributes, including font size for feature labels, feature thickness, gap width between transcripts, and feature highlight properties. These controls allow the user to highlight specific genes while deemphasizing others and produce more compact views in regions of high density. Combined with the image download function, they are also useful for producing images for publication.

\section{Linking and bookmarking}

MGV supports many parameters in the URL used to link to it, and it continually updates the browser's address bar to reflect the user's current view. At any point, the user can bookmark the view or copy/paste the URL into a link. As well, the browser's back/forward buttons work to undo/ redo navigation changes. 


\section{Gene lists}

MGV supports a lightweight notion of lists, which are simply collections of canonical feature IDs. Features in a list can be highlighted in the display, the display can be limited to just the features in a list, or list features can be removed from the display. Lists can be created by entering IDs, by selecting features in the display, and by querying biological annotations such as disease and phenotype associations. For example, a researcher can create a list of mouse genes associated with Parkinson's disease based on expertly curated information from MGD, display those genes on a genome overview map, and then select one or more genes from the genome map to explore in detail across strains.

\section{Sequence download}

A powerful feature of MGV is the ability to select and download sequences from displayed genomes. Selecting sequences adds descriptors to a sequence cart which functions like an online shopping cart. From the sequence cart, researchers can download the sequences for their selected genome features in FASTA format to a file, browser tab, or to the clipboard. Because the sequence cart contains only descriptors, there is no limit to the number of sequence features listed there. However, a single download operation is limited to a maximum of $100 \mathrm{MB}$ and 4000 sequences.

To select genomic, transcript or CDS (coding sequence) sequences for a specific gene (and optionally for all its homologs) a user can open the gene's context menu (right click on the gene) and choose the appropriate option. By default, CDS sequences are added with amino acid translation, but this setting can be disabled in the Sequence Cart. MGV users can also select sequences for genomic regions of interest by option-dragging with the mouse. If the scroll lock (lock icon in control area) is on, the same regions are selected from all the displayed genomes; otherwise, the selection applies only to the specific genome. The direction that the user drags the mouse determines whether reverse complementation is initially active (right to left) or inactive (left to right).

\section{Image download}

The user can download an image of the current view by clicking the camera icon in the main navigation panel. By default, the image is downloaded in PNG (portable network graphics) format. Shift-clicking the camera icon downloads the image in SVG (scalable vector graphics) format. Compared to a direct screen capture, the image download function returns the entire view, including sections that may be scrolled out of view. As well, the SVG option allows the image to be rendered at any desired resolution. User control over font size, track spacing, and other display parameters also aid in producing images for publication.

\section{Use cases}

The primary driver for the development of MGV was to allow researchers to visualize and explore genome features and their organization across different mouse genomes. The use cases described below are examples for two of the main uses of MGV: exploring annotation similarities and differences across the genomes of multiple mouse strains and comparing the organization of homologous genome features from different organisms.

\section{Display and explore genome-specific annotation differences}

The ease with which sequences (genomic, cDNA, CDS, reverse complement, etc.) can be accessed with MGV greatly simplifies the comparative genome annotation analysis needed to identify annotations that are in one genome and not another and to determine if the condition represents true strain-specific differences or is the result of incomplete genome assemblies and/or missing annotations (Lilue et al. 2018). The MGV display align feature provides a comparative view of a given genome feature to identify similarities and differences between genomes for that feature. While not a multiple sequence alignment tool, the MGV display alignment functionality centers the view on a selected feature, aligns corresponding features at their $5^{\prime}$ end across the genome tracks for all the user-selected genomes, and dynamically scales each genome so the selected feature occupies approximately the same span. The sequences that underlie the genome features are easily accessed and saved using MGV's sequence cart and subsequently submitted to external sequence alignment and analysis tools.

For example, aligning the display of annotations across multiple strains for the Evalc gene (MGI:1918217) shows that this gene has been annotated in several mouse strains, but not in 129S1/SvImJ and other strains (Fig. 3A). The expression of Evalc (a novel Slit receptor gene) is expressed in axons of developing forebrain and spinal cord and likely plays a role in Slit/Robo mediated axon guidance (James et al. 2013). We used MGV's sequence extraction functions in conjunction with the National Center for Biotechnology Information (NCBI) Splign tool (Kapustin et al. 2008) to further investigate the putatively missing Evalc annotations. The Splign tool efficiently compares cDNA to genomic sequence, provides useful displays of sequence alignments that highlight differences, and overlays CDS translations on the alignments. We used MGV's Sequence 


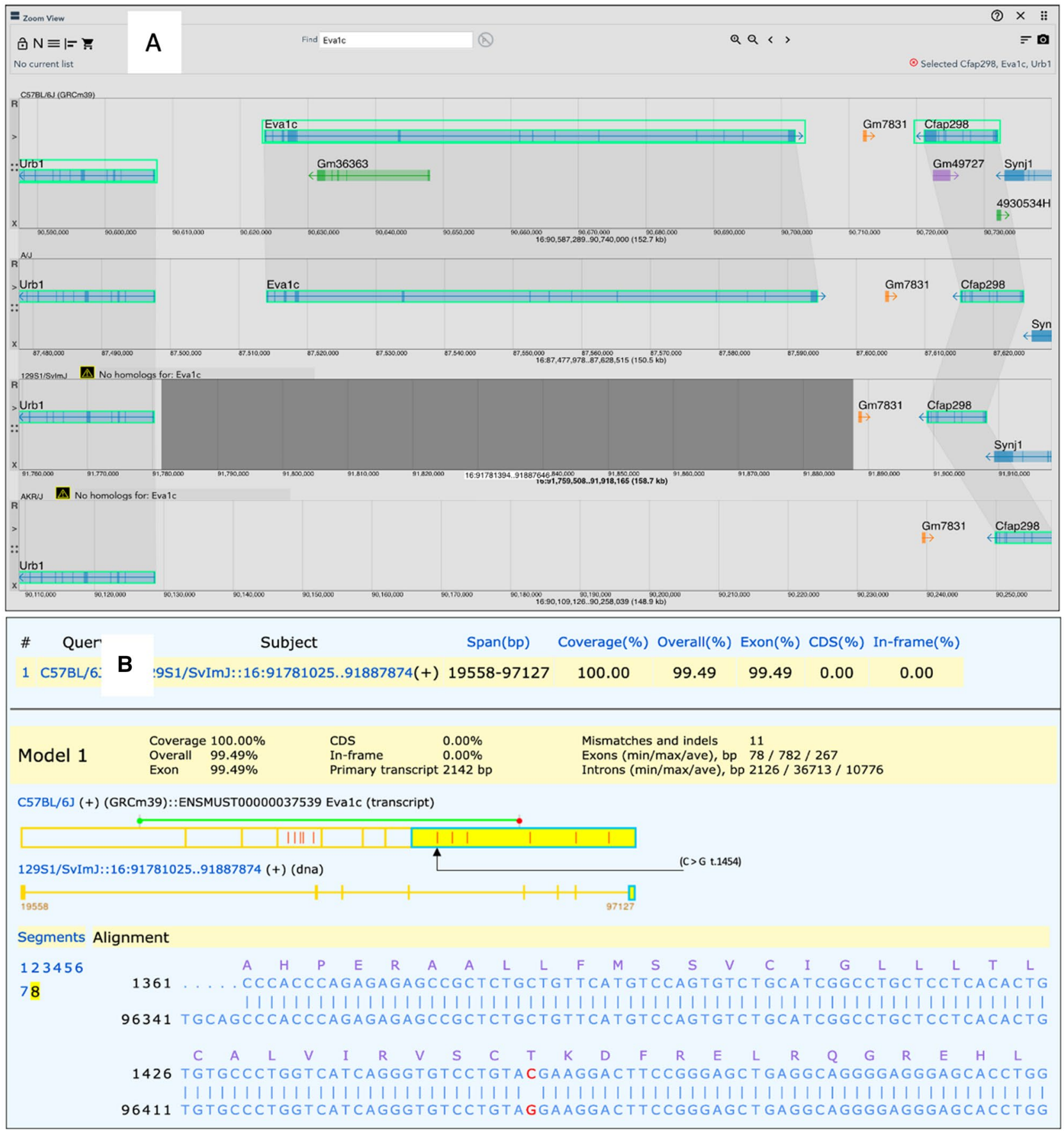

Fig. $3 \mathrm{MGV}$ can aid in the evaluation of missing annotations in different mouse strains. A A region of Chr16 is shown flanked by the Urb1 and Cfap298 genes (MGI:2146468 and MGI:1915251, respectively). Vertical connections (swim lanes) between features indicate equivalent annotations in those genomes. The Evalc gene is annotated in several mouse strains (C57BL/6J, and A/J shown), but not in others (129S1/SvImJ, AKR/J shown). B NCBI Splign tool output using sequences from MGV's Sequence Cart function showing that
Evalc is present in the $129 \mathrm{~S} 1 / \mathrm{SvImJ}$ genome, but is not annotated due to incomplete annotation of the genome assembly. The $5^{\prime}$ end of the 8th cDNA/genomic alignment is shown, with conceptual translation. Differences between aligned sequences are indicated by red vertical bars in the transcript segments. All sequence variants in the CDS are synonymous, except for a $\mathrm{C}>\mathrm{G}(\mathrm{B} 6>129)$ non-synonymous transversion (Thr $>\mathrm{Arg}$ ) at position 1454 in the transcript (black arrow) 
Cart function to download genomic sequence from strains that either lacked or included the Evalc annotation and then performed an alignment with an Evalc transcript sequence from C57BL/6J (ENSMUST00000037539) using Splign. The alignments revealed that the Evalc gene is, in fact, present in the 129S1/SvImJ genome (Fig. 3B). We also investigated coding sequence variation in Evalc between C57BL/6J and the strains with missing Evalc annotations. The majority of the CDS region variation between the C57BL/6J Evalc transcript and the genomic sequences for 129S1/SvImJ are synonymous substitutions. Similar results were observed for other strains with apparent missing Evalc annotation (AKR/J, CAST/EiJ, CBA/J, DBA/2J, LP/J, PWK/ $\mathrm{PhJ}$ and SPRETUS/EiJ; data not shown). The number of SNPs observed between the C57BL/6J Evalc sequence and strains missing the Evalc annotation is higher on average than between strains with the annotation (8-25 SNPs for strains without the Evalc annotation compared to 0-7 SNPs for strains with the annotation). For exons contained in the C57BL/6J Evalc transcript used, in no strain was a nonsense substitution observed and only a single conserved indel was observed in the $5^{\prime}$-UTR for two strains that lack the Evalc annotation (DBA/2J and $\mathrm{PWK} / \mathrm{PhJ})$. As expected, Evalc annotations from wild-derived strains, CAROLI/EiJ and PAHARI/EiJ, show more variation when compared to C57BL/6J Evalc (46 SNPs/4 UTR indels, 99 SNPs/6 UTR indels, respectively). That none of the variation observed in any of these strains interrupts the Evalc CDS region strongly supports the suggestion that this gene is functional in these strains and that the missing Evalc gene is a matter of incomplete annotation.

A second case study for comparative genome annotation assessment using MGV centers on the Femla gene (MGI:1335089) on chromosome 17 (Fig. 4). The Femla gene encodes an ankyrin repeat-containing protein that interacts with prostaglandin E2 receptor 4 (Ptger4; MGI:104311) to inhibit NF- $\mathrm{KB}$-mediated inflammation in macrophages (Minami et al. 2008). We investigated the missing Femla annotations on chromosome 17 in several mouse strains using the same approach for Evalc described above using MGV's sequence cart function to see if these strain differences were real and potentially biologically significant or due to technical issues. The analysis revealed that the missing Femla annotations in several strains are not due to strain

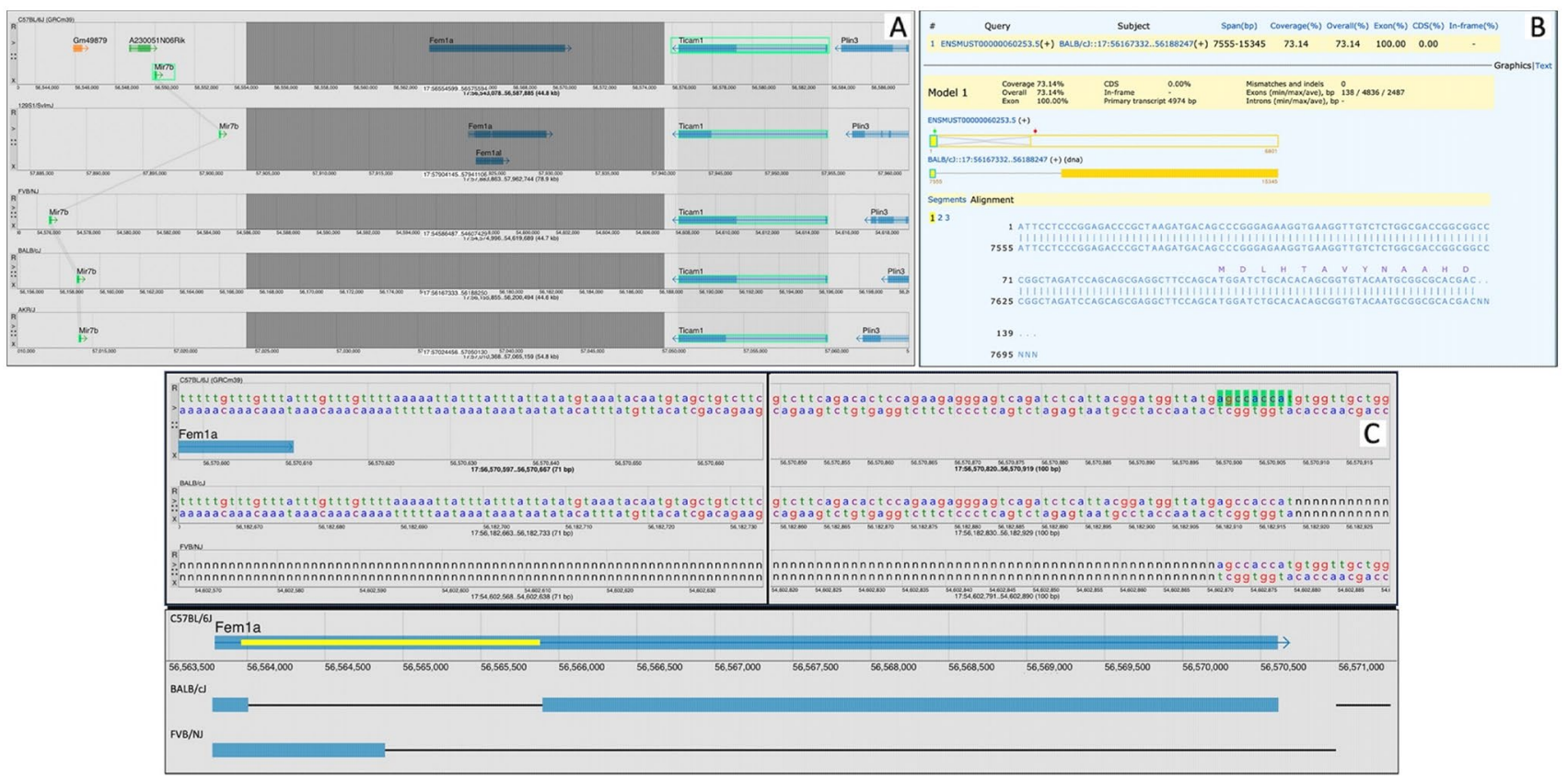

Fig. 4 MGV can reveal genome issues with genome assemblies. A The Femla region on $\mathrm{Chr} 17$ is shown for genomes of strains C57BL/6J, 129S1/SvImJ, FVB/NJ, BALB/cJ, and AKR/J. The region shown is bounded by the Ticaml and Mir7b genes (highlighted) to illustrate that the Femla region is bounded by common annotations in all strains. Femla annotations are present in C57BL/6J and 129S1/ SvImJ, but absent from the other strains shown. B NCBI Splign alignment of C57BL/6J Fem1a transcript ENSMUST00000060253 against the BALB/cJ genomic sequence flanked by Ticaml and Mir7b. C Femla region gaps in the BALB/cJ and FVB/NJ genomes on Chr17. Top panel: base pair-level resolution in the MGV for a region at the $3^{\prime}$ end of the Fem1a gene in C57BL/6J, BALB/cJ, and $\mathrm{FVB} / \mathrm{NJ}$. The three genomes were manually aligned on a $9 \mathrm{bp}$ sequence that borders opposing gaps in BALB/cJ and FVB/NJ (green highlight). Vertical black bar represents $\sim 182$ bp excluded for display. Bottom panel: schematic overview of sequence gaps in the Fem1a gene region of BALB/cJ and FVB/NJ strain genomes. Full C57BL/6J Fem1a transcribed region shown with GRCm39 coordinates and CDS (yellow bar). Relative locations of sequence gaps in BALB/cJ and $\mathrm{FVB} / \mathrm{NJ}$ show as thin black lines 
divergence but are instead linked to sequence gaps in the current genome assemblies on chromosome 17 (Fig. 4B,C).

While most of the available mouse genomes have the Femla gene located on chromosome 17, this gene is currently annotated on chromosome 11 in strains BALB/cJ and FVB/NJ. Most strains have a Femla-related gene (Femlal; MGI: 2441689) annotated on chromosome 11, but for several strains (129S1/SvImJ, C57BL/6NJ, LP/J, WSB/EiJ) the Femlal gene is annotated to chromosome 17 (data not shown). The sequence gaps in the genome assemblies identified using the MGV on chromosome 17 will need to be resolved before the relationships between Femla-related sequences on chromosomes 11 and 17 across different mouse strains can be fully and accurately characterized.

The strain distribution of the $C w c 22$ gene (CWC22 spliceosome-associated protein; MGI:2136773) illustrates a difference in annotation across mouse strains that is the result of evolutionary divergence as opposed to technical issues with genome sequence quality and completeness. $C w c 22$ is annotated in all of the available mouse strain genomes with the exception of the Spretus/EiJ genome (Fig. 5). The gene is contained within the $\mathrm{R} 2 \mathrm{~d}$ segmental duplication region of the mouse genome (Morgan et al. 2016). We aligned a transcript of the $C w c 22$ gene from C57BL/6J to the Spretus/EiJ genome using Splign and revealed the existence of a remnant of the gene in Spretus with significant sequence decay (Fig. 5). Future updates of genome features in the Spretus/EiJ genome should include the annotation of a Cwc22 pseudogene.

\section{Display and explore genome annotations across organisms}

MGV supports the exploration of the genome context of orthologous genes from different organisms. For example, there are two mouse orthologs of the human THOC2 gene (THO Complex 2; HGNC:19073): Thoc2 (MGI:2442413) and Thoc2l (MGI:3040669) located on chromosomes X and 5, respectively (Fig. 6). The human THOC2 gene is associated with the disease X-linked intellectual disability-short stature-overweight syndrome (DOID:0112056; OMIM \#300957). Aligning the MGV display on human THOC2 produces a useful split view of the two mouse orthologs (Fig. 6). MGV does this automatically for all orthologs of a selected feature in the genomes displayed where orthologs in a given comparison genome are present on different chromosomes or located outside of the rendered view. It is clear from the genome context that the mouse chromosome $\mathrm{X}$ locus is in a region of conserved synteny with respect to human THOC (Fig. 6A).

An amino acid percent identity matrix generated by downloading protein sequences to MGV's sequence cart and sending them to Clustal Omega (Sievers et al. 2011) reveals that the mouse THOC2 protein is $97.5 \%$ identical to the human protein (Fig. 6B). The mouse THOC2L protein is also highly similar to human THOC2 $(90.6 \%$ amino acid identity) (Fig. 6B). In fact, the mouse THOC2L protein is more similar to human THOC2 than it is to mouse THOC2. The design of genome editing reagents to generate mouse models of X-linked intellectual disability-short stature-overweight syndrome should consider the high sequence similarity of mouse Thoc $2 l$ gene on chromosome 5 . While the THOC 2 case described above is a relatively simple example, MGV is designed to easily visualize and interact with multiple orthologs at one time. As each genome section containing an ortholog of the selected feature has independent browsing operations, exploration of genome context in each segment and generation of customized views are possible.

Because MGV supports dynamic scaling of multiple genome regions and has numerous display control options, it is very well suited for comparative analyses of multigene families (Fig. 7). For example, the cytochrome P450 family of monooxygenases are central to the metabolism of endogenous compounds and also for drug metabolism. The CYP3A subfamily catalyzes the largest fraction of the many P450-mediated metabolic clearance reactions known (Rendic and Guengerich 2015). Mouse models are important in predicting efficacy and potential human side effects of novel therapeutics, but effective model depiction must consider differences in the $\mathrm{P} 450$ gene repertoires between human and mouse (Henderson et al. 2019). MGV provides homology-based views of annotated gene family members based on strict definitions of orthologs from the Alliance of Genome Resources (Alliance of Genome Resources 2019; Alliance of Genome Resources Consortium 2019). An MGV view of relationships between the mouse Cyp3a25 gene (MGI:1930638) and orthologs in human and rat is shown in Fig. 7B. Human orthologs of mouse Cyp $3 a 25$ include the CYP3A4 (HGNC: 2637) and CYP3A5 (HGNC: 2638) genes, known to account for over $30 \%$ of the metabolic reactions involving marketed and developmental therapeutic drugs (Rendic and Guengerich 2015). MGV allows users to toggle between strict orthologous connections between gene family members and a view of inferred paralogous relationships (Fig. 7A). The paralog option provides a view of the complexity of this gene family in the mouse genome, and MGV provides easy access to sequence data from each family member for further analysis to aid in the design of humanized mouse models in those cases where multiple mouse genes need to be replaced with their human gene equivalents (Henderson et al. 2019). 

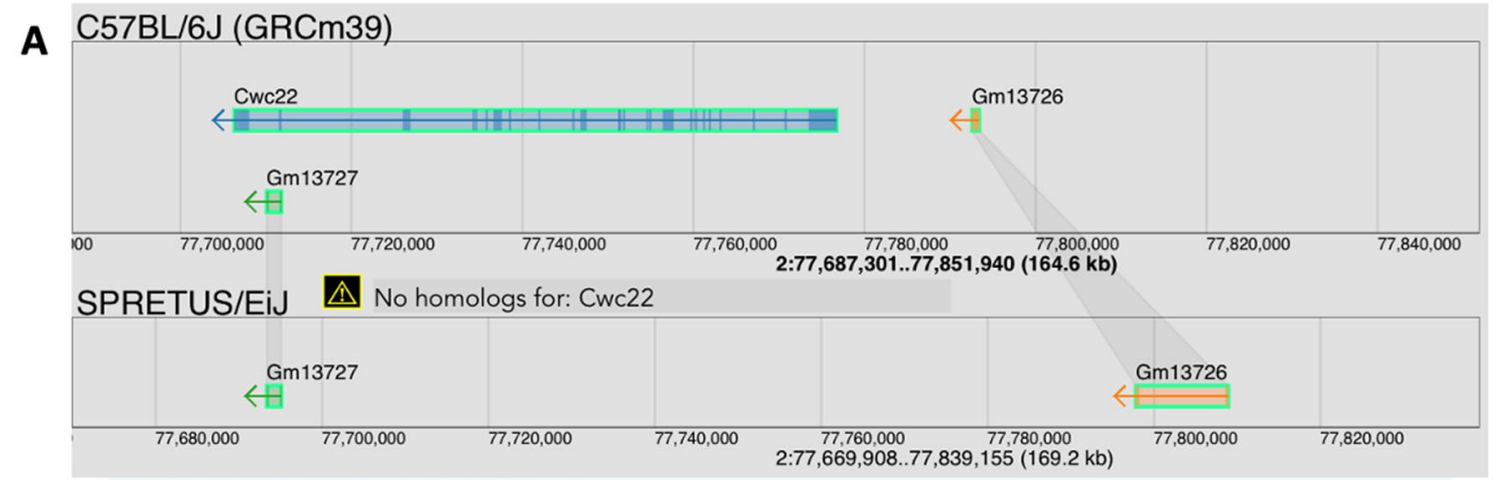

B

C57BL/6] (+) (GRCm39)::MGI_C57BL6]_2136773_GRCm39_composite Cwc22 (transcript)

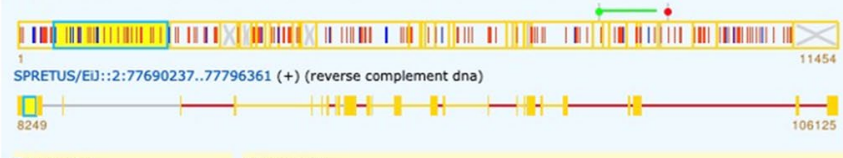

Segments Alignment

123456789910 11121314151617181920 21222324252627282930 31323334353637383940 41

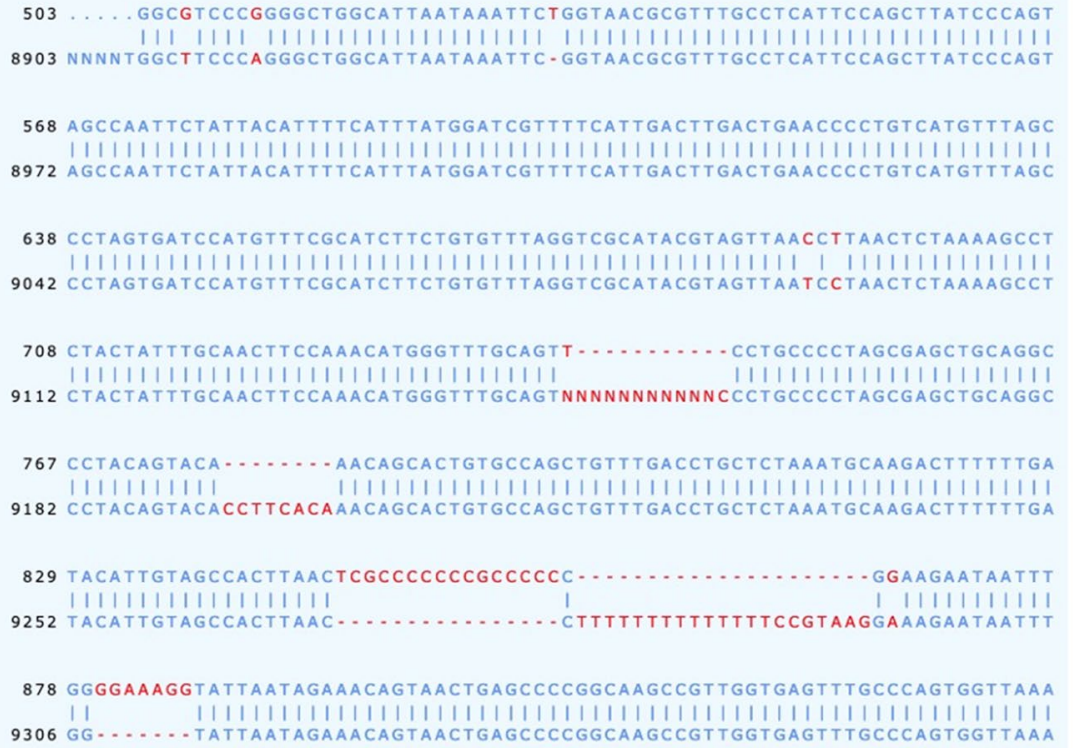

Fig. 5 A MGV aligned view of the $C w c 22$ gene showing the missing annotation in Spretus/EiJ. B NCBI Splign alignment of the $C w c 22$ transcript from C57BL/6J to the Spretus/EiJ genome reveals a remnant of the $C w c 22$ gene in Spretus with severe evolutionary decay in the sequence

\section{Methods}

\section{Implementation and availability}

MGV is accessible from the mouse genome informatics (MGI) web site (http://www.informatics.jax.org/mgv/). By default, the viewer provides access to genome features for 19 mouse genomes and annotations from the reference genomes for human (H. sapiens), fruit fly (D. melanogaster), zebrafish (D. rerio), nematode (C. elegans), rat ( $R$. norvegicus), and yeast (S. cerevisiae). For the mouse reference genome, the annotations for the GRCm38 (mm10) and GRCm39 (mm11) assemblies are available.
MGV comprises two software components: the viewer itself, a JavaScript application running in the browser, and the scripts (Python and shell) for preparing the data files used by the viewer. Both software components are available at GitHub: https://github.com/mgijax/mgv and https:// github.com/mgijax/mgv_data, respectively.

The viewer is a single-page JavaScript web app written with the Vue.js reactive framework. A reactive framework (angular and react are two other examples) simplifies web applications by providing features such as template-based rendering and component definition/composition combined with reactivity, wherein the rendered scene automatically updates to reflect changes in the data. These features are 


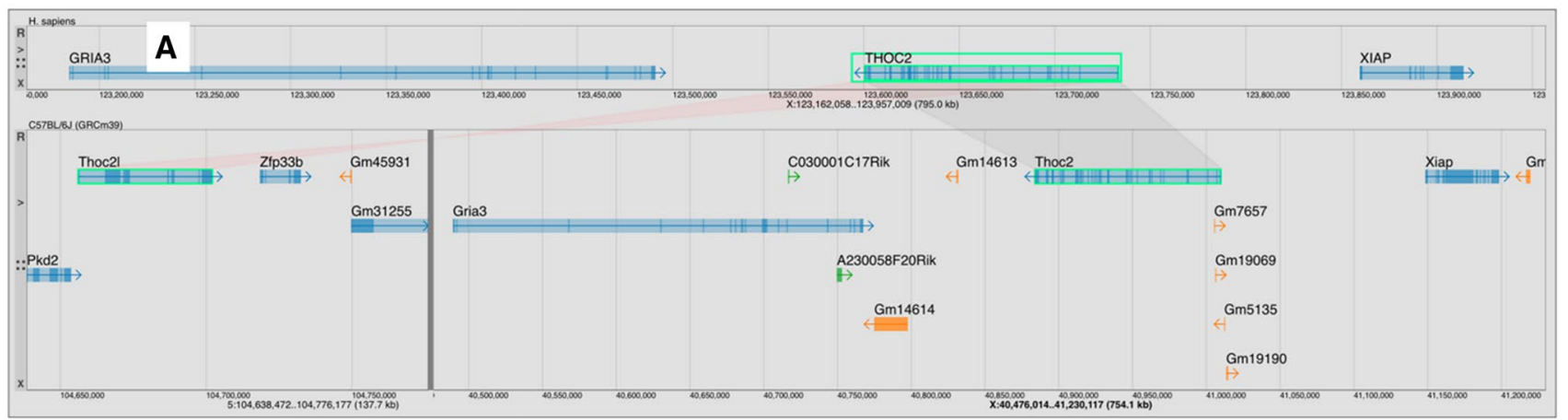

\begin{tabular}{|l|r|r|r|}
\hline & THOC2_HUMAN & ThOc2_MOUSE & Thoc2I_MOUSE \\
\hline THOC2_HUMAN & 100 & 97.49 & 90.55 \\
\hline Thoc2_MOUSE & 97.49 & 100 & 89.73 \\
\hline Thoc2l_MOUSE & 90.55 & 89.73 & 100 \\
\hline
\end{tabular}

Fig. 6 A MGV aligned view of the human THOC2 gene on human Chr X and its two mouse orthologs, Thoc 2 and Thoc2l, on mouse Chr X and Chr 5, respectively. MGV automatically splits the view to show all orthologs of the selected feature in the compared genomes (dark vertical line in the C57BL/6J genome). Relative transcriptional orientation between orthologs is indicated by the color of the swim lanes (light gray or light red for same and

used throughout the viewer code, from organizing the page structure down to rendering features in a genomic region.

Genomic regions are rendered as SVG (scalable vector graphics), a widely used standard language for drawing 2D scenes on the Web. From a programming standpoint, SVG offers several attractive features, especially combined with templates and reactivity. Drawing a genomic region becomes more declarative, and the application's code can focus on data manipulation. The main issue with using SVG is rendering performance for large scenes when there are many thousands of nodes. MGV provides ways for the user to adjust the amount of rendered detail but in general, performance degradation may be noticeable for large regions and/or many genomes.

The mgv_data repository provides the scripts to build the "back end" which serves data to the viewer at runtime. By default, these scripts are configured to build the same data sets being served by the MGV instance deployed at MGI, i.e., the genome assemblies and gene model annotations for nineteen inbred strains of mice and six non-mouse organisms. Customizing the build for other organisms would be relatively straightforward, especially for those available at Ensembl or NCBI. At runtime, the back end comprises many static files (GFF3, plain text, and JSON) and one Python CGI script. Most data requests from MGV are for the static files, while sequence retrievals are served by the CGI. opposite orientations, respectively). B Amino acid percent identity matrix between human THOC2 (NP_001075019), mouse THOC2 (ENSMUSP00000044677), and mouse THOC2L (ENSMUSP00000130629), produced using Clustal Omega showing that the protein produced by the $T H O C 2 L$ paralog has greater sequence similarity to human THOC2 than to mouse THOC2

\section{Summary and future directions}

As more complete genomes become available for the laboratory mouse and other species of mouse, the ability to compare genome features and genome organization across different individuals and strains will become increasingly important for investigations into how genome differences relate to differences in biological function. MGV is a versatile and extendable software tool with powerful interactive features to support comparative genomics research. The ability for researchers to highlight genome features across different genomes is one of the features of the software that sets it apart from other available genome browsers. As illustrated in the examples provided in this report, MGV supports visualization and analysis functions that support the evaluation of annotation differences across strains to determine if they are true differences or are due to technical issues with the current genome assemblies.

Several features are under development for future releases of MGV. One of the major enhancements planned for MGV is support for comparisons of the structure of individual genes (e.g., introns, exons, transposable elements, endogenous retroviral insertions sites, etc.) across different strains of mice. As described in Keane et al. (Keane et al. 2011), differences in gene structure across mouse strains have been documented for many genes including, $\operatorname{Prdm} 9, \operatorname{Mcm} 9$, and Poli. Allowing researchers to quickly compare gene structures will be important for identifying putative functional 


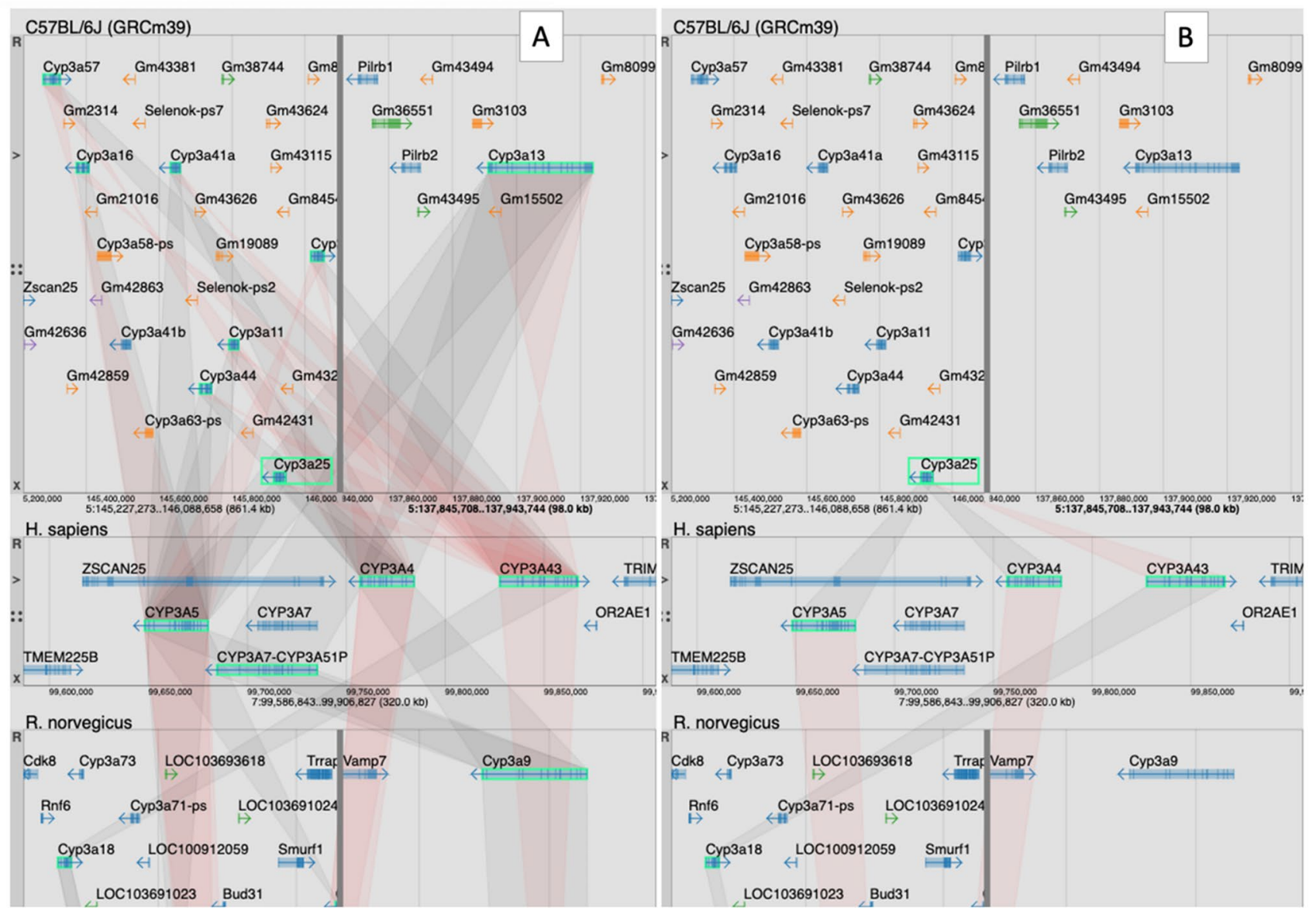

Fig. 7 Screenshot of MGV showing mouse Cyp3a25 in C57BL/6J, human, and rat with $(\mathbf{A})$ and without $(\mathbf{B})$ inferred paralogs displayed. MGV uses data from the Alliance of Genome Resources (https://allia ncegenome.org) for non-mouse gene models and orthology assertions. The color of the swim lane connecting orthologs indicate if the

variants in the genomes of different mouse strains and species. Future versions of MGV will also support upload of private annotated genomes and the integration and visualization of gene expression and any other data type that can be represented meaningfully using standard genome annotation and display formats such as GFF, WIG, and BED (http:// genome.ucsc.edu/FAQ/FAQformat). The current inferences of paralogs used in MGV will be replaced with paralog assertions provided by the Alliance of Genome Resources when these become available. We will also extend the gene list functionality of MGV to include annotations for model organisms other than the laboratory mouse by integrating The Alliance of Genome Resources recently released AllianceMine database as the annotation source. Finally, we will add the new rat genome assembly (mRatBN7.2; https://tbmkl.xyz/5b531c0a1a4d134a5513100e441e4f0c) to support comparing annotations on this recently released assembly to the previous one for rat (Rnor_6.0). orientation of the genome feature is in the same (light gray) or opposite (light red) orientation. Relationships of genome features across organisms can be complex due to biology and/or genome annotation and assembly issues

Acknowledgements The authors thank the members of the mouse genome database (MGD) Scientific Advisory Board for testing the MGV software and providing valuable feedback. The authors thank the reviewers of the manuscript for their constructive comments on the first version of the manuscript.

Author contributions CJB conceived of the software and its basic functions. JER wrote the code. RMB tested the software and contributed to the design. All authors contributed to the final design and functional properties of the software and to the writing of the manuscript.

Funding This work was supported by National Institutes of Health (NIH) Grants U24 HG000330 and U24 HG10859 to CJB.

Data availability MGV is accessible from the mouse genome informatics (MGI) web site (http://www.informatics.jax.org/mgv/).

Code availability The software for the MGV viewer and the scripts for preparing the data files used by the viewer are available at GitHub: https://github.com/mgijax/mgv and https://github.com/mgijax/mgv_ data, respectively. 


\section{Declarations}

Conflict of interest The authors have no relevant financial or non-financial interests to disclose.

Open Access This article is licensed under a Creative Commons Attribution 4.0 International License, which permits use, sharing, adaptation, distribution and reproduction in any medium or format, as long as you give appropriate credit to the original author(s) and the source, provide a link to the Creative Commons licence, and indicate if changes were made. The images or other third party material in this article are included in the article's Creative Commons licence, unless indicated otherwise in a credit line to the material. If material is not included in the article's Creative Commons licence and your intended use is not permitted by statutory regulation or exceeds the permitted use, you will need to obtain permission directly from the copyright holder. To view a copy of this licence, visit http://creativecommons.org/licenses/by/4.0/.

\section{References}

Alliance of Genome Resources C (2019) The Alliance of Genome Resources: Building a Modern Data Ecosystem for Model Organism Databases. Genetics 213:1189-1196

Alliance of Genome Resources Consortium T (2019) Alliance of genome resources portal: unified model organism research platform. Nucleic Acids Res 48:D650-D658

Baldarelli RM, Smith CM, Finger JH, Hayamizu TF, McCright IJ, Xu J, Shaw DR, Beal JS, Blodgett O, Campbell J, Corbani LE, Frost PJ, Giannatto SC, Miers DB, Kadin JA, Richardson JE, Ringwald M (2021) The mouse gene expression database (GXD): 2021 update. Nucleic Acids Res 49:D924-D931

Blake JA, Baldarelli R, Kadin JA, Richardson JE, Smith CL, Bult CJ, Mouse Genome Database G (2020) Mouse genome database (MGD): knowledgebase for mouse-human comparative biology. Nucleic Acids Res 49:D981-D987

Henderson CJ, Kapelyukh Y, Scheer N, Rode A, McLaren AW, MacLeod AK, Lin D, Wright J, Stanley LA, Wolf CR (2019) An extensively humanized mouse model to predict pathways of drug disposition and drug/drug interactions, and to facilitate design of clinical trials. Drug Metab Dispos 47:601-615

Iraqi FA, Churchill G, Mott R (2008) The collaborative cross, developing a resource for mammalian systems genetics: a status report of the wellcome trust cohort. Mamm Genome 19:379-381

James G, Foster SR, Key B, Beverdam A (2013) The expression pattern of EVA1C a novel slit receptor is consistent with an axon guidance role in the mouse nervous system. PLoS One 8:e74115

Kapustin Y, Souvorov A, Tatusova T, Lipman D (2008) Splign: algorithms for computing spliced alignments with identification of paralogs. Biol Direct 3:20

Keane TM, Goodstadt L, Danecek P, White MA, Wong K, Yalcin B, Heger A, Agam A, Slater G, Goodson M, Furlotte NA, Eskin E, Nellaker C, Whitley H, Cleak J, Janowitz D, Hernandez-Pliego
P, Edwards A, Belgard TG, Oliver PL, McIntyre RE, Bhomra A, Nicod J, Gan X, Yuan W, van der Weyden L, Steward CA, Bala S, Stalker J, Mott R, Durbin R, Jackson IJ, Czechanski A, Guerra-Assuncao JA, Donahue LR, Reinholdt LG, Payseur BA, Ponting CP, Birney E, Flint J, Adams DJ (2011) Mouse genomic variation and its effect on phenotypes and gene regulation. Nature 477:289-294

Lilue J, Doran AG, Fiddes IT, Abrudan M, Armstrong J, Bennett R, Chow W, Collins J, Collins S, Czechanski A, Danecek P, Diekhans M, Dolle DD, Dunn M, Durbin R, Earl D, FergusonSmith A, Flicek P, Flint J, Frankish A, Fu B, Gerstein M, Gilbert J, Goodstadt L, Harrow J, Howe K, Ibarra-Soria X, Kolmogorov M, Lelliott CJ, Logan DW, Loveland J, Mathews CE, Mott R, Muir P, Nachtweide S, Navarro FCP, Odom DT, Park N, Pelan S, Pham SK, Quail M, Reinholdt L, Romoth L, Shirley L, Sisu C, Sjoberg-Herrera M, Stanke M, Steward C, Thomas M, Threadgold G, Thybert D, Torrance J, Wong K, Wood J, Yalcin B, Yang F, Adams DJ, Paten B, Keane TM (2018) Sixteen diverse laboratory mouse reference genomes define strain-specific haplotypes and novel functional loci. Nat Genet 50:1574-1583

Minami M, Shimizu K, Okamoto Y, Folco E, Ilasaca ML, Feinberg MW, Aikawa M, Libby P (2008) Prostaglandin E receptor type 4-associated protein interacts directly with NF-kappaB1 and attenuates macrophage activation. J Biol Chem 283:9692-9703

Morgan AP, Holt JM, McMullan RC, Bell TA, Clayshulte AM, Didion JP, Yadgary L, Thybert D, Odom DT, Flicek P, McMillan L, de Villena FP (2016) The evolutionary fates of a large segmental duplication in mouse. Genetics 204:267-285

Rendic S, Guengerich FP (2015) Survey of human oxidoreductases and cytochrome P450 enzymes involved in the metabolism of xenobiotic and natural chemicals. Chem Res Toxicol 28:38-42

Sievers F, Wilm A, Dineen D, Gibson TJ, Karplus K, Li W, Lopez R, McWilliam H, Remmert M, Soding J, Thompson JD, Higgins DG (2011) Fast, scalable generation of high-quality protein multiple sequence alignments using Clustal Omega. Mol Syst Biol 7:539

Thybert D, Roller M, Navarro FCP, Fiddes I, Streeter I, Feig C, Martin-Galvez D, Kolmogorov M, Janousek V, Akanni W, Aken B, Aldridge S, Chakrapani V, Chow W, Clarke L, Cummins C, Doran A, Dunn M, Goodstadt L, Howe K, Howell M, Josselin AA, Karn RC, Laukaitis CM, Jingtao L, Martin F, Muffato M, Nachtweide S, Quail MA, Sisu C, Stanke M, Stefflova K, Van Oosterhout C, Veyrunes F, Ward B, Yang F, Yazdanifar G, Zadissa A, Adams DJ, Brazma A, Gerstein M, Paten B, Pham S, Keane TM, Odom DT, Flicek P (2018) Repeat associated mechanisms of genome evolution and function revealed by the Mus caroli and Mus pahari genomes. Genome Res 28:448-459

Zhu Y, Richardson JE, Hale P, Baldarelli RM, Reed DJ, Recla JM, Sinclair R, Reddy TB, Bult CJ (2015) A unified gene catalog for the laboratory mouse reference genome. Mamm Genome 26:295-304

Publisher's Note Springer Nature remains neutral with regard to jurisdictional claims in published maps and institutional affiliations. 